\title{
Wireless Infrastructure in a District Heating Substation
}

\author{
Jan van Deventer, Jonas Gustafsson, Jerker Delsing, Jens Eliasson \\ Luleå University of Technology \\ S-97187 Luleå, Sweden \\ Email:\{jan.van.deventer, j.gustafsson, jerker.delsing, jens.eliasson\} @1tu.se
}

\begin{abstract}
A new wireless sensor and actuator network architecture for a district heating substation is presented. The implementation allows for new control algorithms to save substancial amounts of energy and therefore reduce waste and indirectly $\mathrm{CO}_{2}$ emissions. The wireless network uses TCP/IP all the way to the sensor level, which can enable a standardized Service Oriented Architecture and the possibility to upgrade software of each node remotely over the Internet. New diagnostic services are also available to improve service and maintenance.
\end{abstract}

\section{INTRODUCTION}

Pervasive technologies can be a blessing if they contribute to society without increasing stress or frustration to the population. An area where pervasive technology can make a substantial improvements is energy management and control within district heating. For the purpose, we here present a control and communication architecture based on wireless sensor/actuator network (WSN). The architecture is implemented through a wireless network of sensors and actuators to control the energy transfer from a district heating distribution network to a building while promoting the potential of Service Oriented Architecture (SOA) towards an easy integration and expansion of the pervasive technology.

The approach permits the development of new control strategies in the optimization of energy transfer operation because the system offers more information. Such strategies target an increased cooling of the distribution network, i.e. a higher $\Delta \mathrm{T}$ over the substation. A higher $\Delta \mathrm{T}$ is beneficial for a district heating system regarding increased co-generation of electricity, reduced energy losses, and more efficient utilization of infrastructure investments.

The implementation of new control strategies and services will be much simplified using a SOA based approach [1], [2], [3]. Thus it is important that the WSN:nodes have SOA properties like service discovery and a service description semantics which enables an orchestration of services such that new control strategies can be deployed on-line.

The general objective of the paper is to demonstrate a new control and communication architecture for district heating substation and to show that WSN with SOA is a feasible technology for this architecture.

To outline the paper, we first give a background to district heating and describe the wireless nodes used. We follow by presenting the wireless network and making the case of Service oriented architecture. The subsequent section discusses the implementation of the wireless infrastructure and its functionality.

\section{A. District heating}

District heating is a technology that enables heating, i.e. both space heating and hot tap water, from one single source to many buildings. It is energy efficient when buildings are close together. An analogy is common transportation where a bus that is more efficient than several cars. Quite often the original heat is a byproduct from an industrial process. The industry can recover some of its cost while end users have a lower heat cost, not to mention the benefit to the environment. This is a wining concept when correctly implemented.

The heat is distributed through networks of pipes to commercial buildings, apartment buildings and houses. The distribution network is also known as the primary circuit. In Sweden, its energy is not used directly in the buildings but rather transfered through heat exchangers within a district heating substation located within the serviced building.

A district heating substation transfers the heat energy from the primary circuit to the building's radiator circuit and hot tap water circuit. These circuits are referred as the secondary circuits. Control systems, electronic and mechanical, regulate the flow of the primary circuit through each heat exchanger via respective valves depending on the energy demands of each of the secondary circuits. It is in the substation that the amount of energy transferred is measured for billing purposes.

\section{B. Control and architecture}

The current problem is that these control systems only aim towards comfort but do not pay attention to optimize the performance of the substation. This results in wasting heat energy in the primary circuit. To optimize the system, additional information is needed but which is nowadays not available. With a wireless connection between sensors and actuators, the new infrastructure allows for the optimization as well as new diagnostics and services.

The traditional control and communication architecture is shown in figure 1 . The heat energy measurement is located on the primary side of the substation. The controls of space heating and hot tap water delivery to the house are made from the secondary side of the substation. These two functions are independent and unaware of each other. 


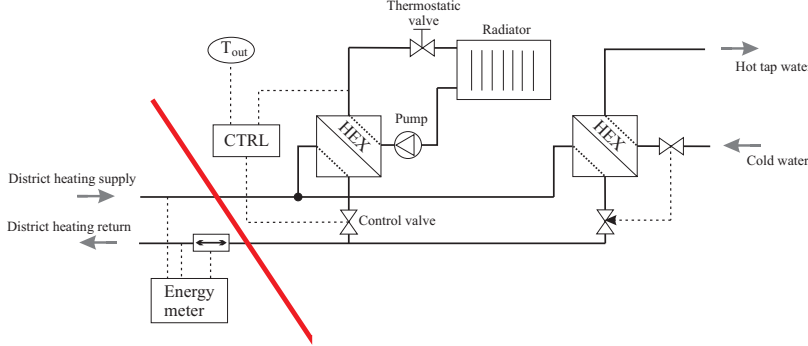

Fig. 1. Traditional control and communication architecture in a district heating substation. Please note the heat measurement at the primary side has no communication to the control at the secondary side (red line).

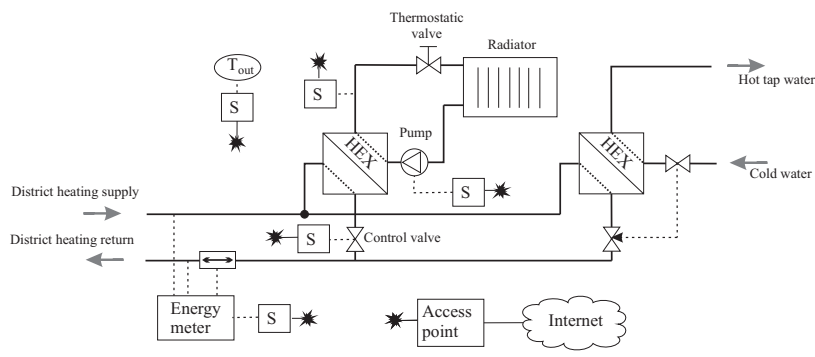

Fig. 2. New control and communication architecture in a district heating substation. Here all sensors, actuators and control devices regardless of location can exchange information.

In the hereby introduced architecture, shown in figure 2, all sensors and actuators are enhanced with or replaced by WSN nodes from which a wireless sensor network based on TCP/IP is established.

This means that the barrier between the primary side with heat metering and the secondary side with heating and hot water control is taken away. This opens up for new measurement possibilities as discussed by Yliniemi [4] and new control strategies as discussed by Wollerstand el.al. [5] and Gustafsson [6].

These new measurement and control strategies have substantial economical potentials. Very rough estimates shows savings of more than 1 million Euro per year when increasing the $\Delta \mathrm{T}$ by $5^{\circ} \mathrm{C}$ in a $760 \mathrm{GWh}$ district heating system.

Using a SOA architecture for the measurement, actuation and control will allow for online changes to measurement and control strategies. Thus providing an architecture that is adaptable to new needs and ideas in a way that is not possible with an architecture based on hard wired systems.

\section{Wireless nodes}

Within the new architecture, each sensor and actuator is associated to a wireless node. We here describe these nodes which we have named Mulle [7], [8].

The Mulle platform is a small embedded system capable of communicating wirelessly with other nodes using TCP/IP over Bluetooth and ZigBee. As all embedded systems, it is able to measure a sensor signal and/or request an action from an actuator. In some cases, the sensor or actuator communication is done serially .

The Mulle features a Renesas M16C/62 micro-controller and in our system, a Bluetooth 2.0 module. It has an on-board $2 \mathrm{MB}$ flash memory, allowing storage of large amounts of sensor data. Flexibility as well as time driven performance are provided by a real-time clock, a temperature sensor, a battery monitor, LEDs and a high density expansion port. The latter is a 60-pin connector that enables connection to analog and digital I/O pins, power supply, and serial communication lines. The compact design occupies a total of $24 \times 26 \times 5 \mathrm{~mm}$.

Power consumption is an issue for the nodes that are not connected to the electric network or associated to actuator. An example in our system are the temperature sensors. They must provide information for a very long duration, which ideally should be years. The current approach is to turn off the Bluetooth module and put the micro-controller to sleep. The power consumption in its lowest sleep mode is only 12 $\mu \mathrm{W}$. The micro-controller is woken up every 15 minutes to measure the temperature. If there is a significant change in temperature, the Bluetooth module is momentarily turned on to communicate the information of the wireless network.

The Mulle software is designed in a modular fashion to allow adaptability to the system and the role of the node within the system. The Mulle can communicate with two of the worlds most widely spread technologies: TCP/IP and Bluetooth. The Mulle uses the lwIP stack, and can communicate using the following protocols: IP, TCP, UDP, DHCP, NTP, and HTTP. The lwBT Bluetooth stack provides support for the following layers: HCI, L2CAP, SDP, BNEP, RFCOMM, and PPP. The Bluetooth Profiles supported are: LAP, DUN, PAN, and SPP.

The use of TCP/IP and Bluetooth enables the Mulle to even utilize Bluetooth-equipped mobile phones as access points to reach the Internet, although, there is no need for that in a district heating application. This allows a Mulle to transmit sensor data directly to users on the Internet without any specialized gateways. The advantage of using TCP/IP at the sensor or actuator level is the clear standardization of the Internet Protocol. This detail enables the infrastructure to be flexible to different sensor and actuator suppliers and opens the door to standardized service oriented architecture.

The nodes can talk to each other or to an access point creating a wireless network. The next subsection describes the implementation of the wireless network.

\section{Wireless network}

The network used in this setup is a IP-based network with DHCP capabilities. The DHCP server is implemented in the Internet access point (c.f. figure 2), which is a Blackfin based embedded computer [9] running $\mu$ Linux. However, any Bluetooth compatible device with the right protocols supported will do.

To be able to run the IP-network on top of Bluetooth, the PANU (Personal Area Network User) Bluetooth profile is used on the Mulles, and the corresponding gateway profile NAP 
(Network Access Point) is used in the Blackfin Internet access point, see figure 3. These profiles utilizes the L2CAP + BNEP Bluetooth protocols to enable an easy integration of TCP/IP network on top, see [10] and [11] for more details.

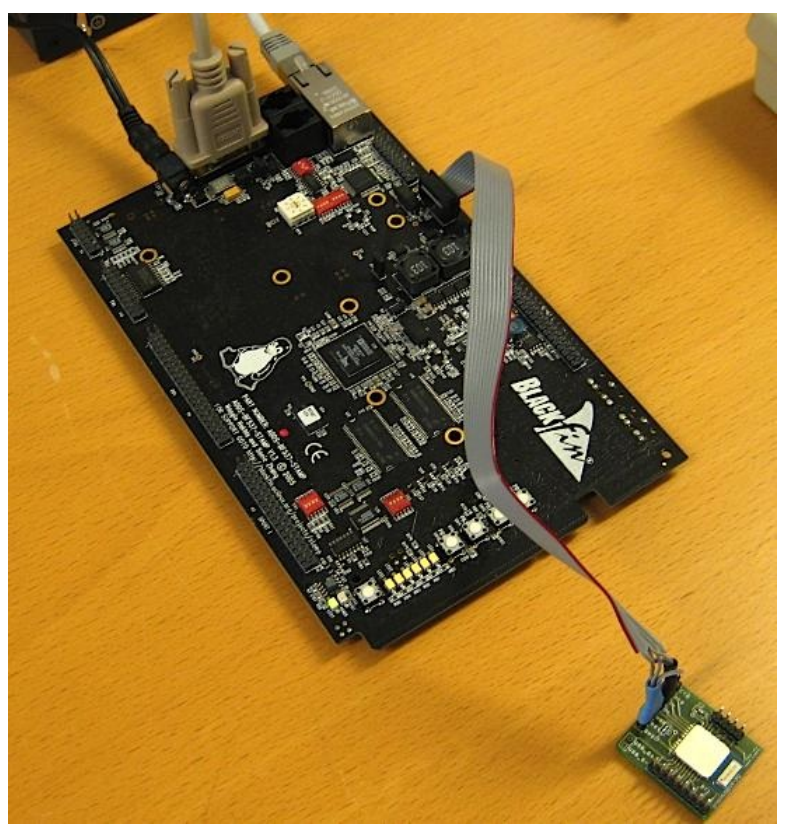

Fig. 3. The Blackfin access point

The control loop is today located in the Blackfin device, but could in the future be distributed between sensor nodes to improve control robustness.

The network setup makes it possible to access all devices (Mulles) in the network from the Internet. This enables the system administrator to reconfigure and update the software in the nodes remotely.

For SOA, the natural solution would be to bring in protocols like DPWS [12], [13] or OPC UA [14]. Due to the constrained resources on the Mulle platform, the current implementation features service discovery mechanisms using Bonjour [15]. A special protocol, OLP v2, which could become a future standard for transmitting sensor data using TCP/IP, has been developed. OLP v2 is used to to send information and requests between all the nodes.

\section{IMPLEMENTATION}

A Mulle node is placed on each actuator, sensor and meter. The nodes are small enough to fit in the electronics of the commercially available actuators. The circulation pump is a Grundfos UPE 25-40 unit, see figure 4. It has it own microcontroller, several sensors such as pressure, and communicates through an RS-485 link using a GENIbus protocol readying the unit for industrial use. The Mulle is the link between the pump and the wireless network. The Mulle can request a given flow and provide to the wireless network information about the radiator circuit.
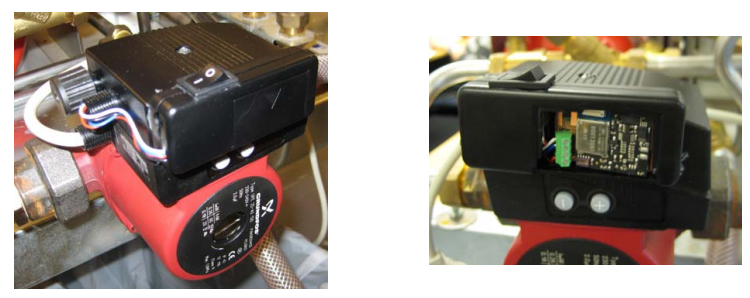

Fig. 4. The pump with belonging wireless node.

The control valve limiting the primary flow that serves the radiator circuit's heat exchanger is a Siemens valve model SQS65, see figure 5. Its control and feedback signals are over the range of 0 to $10 \mathrm{~V}$. The Mulle can request a valve position or measure what the position of the valve informs the wireless network.
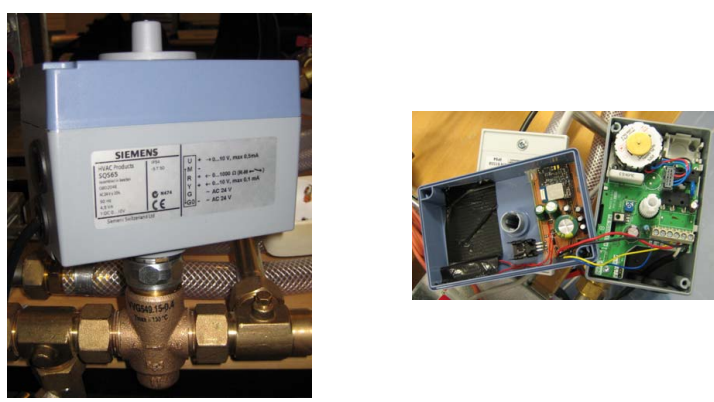

Fig. 5. The valve with its wireless node.

The temperature wireless sensor node uses a external digital temperature sensor from Dallas Semiconductor (DS18B20), see figure 6 . It uses a 1-wire interface to serially communicate with the Mulle. The Mulle itself has an on-board temperature sensor but the external one offers the possibility of measuring the temperature of pipes for example. In the current setting, the unit is used to measure outdoors temperature. This unit is battery powered and therefore makes use of the low power features of the Mulle.

The primary circuit's incoming and outgoing temperatures (with their difference being $\Delta \mathrm{T}$ ) as well as its flow are 

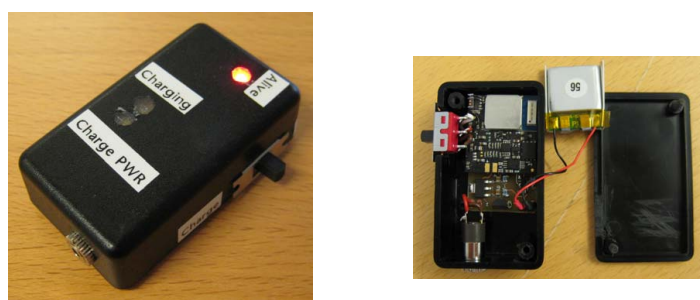

Fig. 6. The battery driven temperature sensor node.

measured by the heat meter, a Kamstrup MultiCal 601, figure 7. The Mulle implements the Kamstrup Meter Protocol (KMP) to serially communicate with the heat meter and is a drop in replacement to the optional communication module for the Kamstrup heat meter.
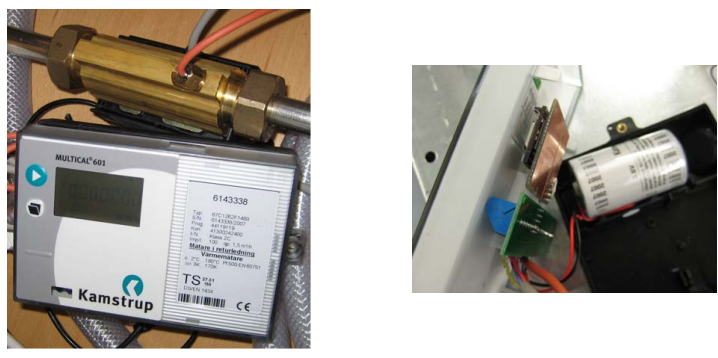

Fig. 7. The Kamstrup heat meter.

\section{A. Benefits}

When taking into account the incoming and outgoing temperatures of the primary circuit in the control of indoor comfort, we can achieve a higher $\Delta \mathrm{T}$ and therefore obtain a better energy transfer. The old architecture did not allow this which results in at least two forms losses. The first one is similar to an electrical short circuit, it allows the energy to go through the house without doing any work although pumps are being used to generate the flow. This also causes inefficiency if the heat is from an electricity co-generating plant (Combined Heat and Power plant). The second form of waste when $\Delta \mathrm{T}$ is not maximized is the distribution losses through the primary network. That is there is large thermal gradient between the transport fluid and the outdoors temperature resulting in higher heat transfer to the environment. By maximizing $\Delta \mathrm{T}$, not only the return temperature will be lowered but the supply temperature can be lowered.

It should therefore be obvious that an efficient system will reduce the amount of wasted energy while maintaining comfort. This will indirectly reduce $\mathrm{CO}_{2}$ emissions for heating purposes which accounts for $30 \%$ of the world's current $\mathrm{CO}_{2}$ [16]. If the heat is an industrial byproduct, then one could argue that it does not matter. However that would be incorrect as any excess heat can be used to serve other building, which use other means of heating.

The new architecture additionally allows improved quality of service and maintenance. For example, if the flow meter, which is a part of the heat meter, detects a flow while the control valves are closed, we can diagnose a leaky valve. The connection to the Internet permits the information to be received immediately by the heat supplier. As an other illustration, the circulation pump is able to measure pressure in the radiator circuit, but this feature was never used. With its wireless communication, the system can now, for instance, send a text message to the home-owner's phone to tell that there is not enough water in the radiator circuit.

\section{CONCLUSION AND FUTURE WORK}

We have developed a new control and communication architecture based on WSN and SOA for district heating substations. The architecture has successfully been implemented in a working substation. The use of TCP/IP and SOA in combination with a simple integration of the WSN node to any sensor, actuator or control device enables simple retrofitting of existing substations and demonstrates a technology for next generation of substation measurement and control.

For the future some of the new control strategies will be implemented in a pilot installation. At a planing stage is a larger pilot study comprising a large number of installations in a complex district heating system. Next then will come work on control strategies which does incorporate the end user and end user interaction with the WSN and SOA based control architecture.

\section{ACKNOWLEDGMENT}

We thank the Swedish District Heating Association for their financial support with the project but also for their involvement and feedback.

\section{REFERENCES}

[1] M. Feike, N. Popova, and A. W. Colombo, "Ami-based production systems: Configuration of sequential process flow and soa-based diagnosis," Piscataway, NJ 08855-1331, United States, 2008, pp. 517 - 524. [Online]. Available: http://dx.doi.org/10.1109/INDIN.2008. 4618155

[2] A. Gaeta, M. Gaeta, A. Smith, I. Djordjevic, T. Dimitrakos, M. Colombo, and S. Miranda, "Design patterns for secure virtual organization management architecture," Piscataway, NJ 08855-1331, United States, 2007, pp. 207 - 216. [Online]. Available: http: //dx.doi.org/10.1109/SECCOM.2007.4550334

[3] V. Herrera, A. Bepperling, A. Lobov, H. Smit, A. Colombo, and J. L. M. Lastra, "Integration of multi-agent systems and service-oriented architecture for industrial automation," Piscataway, NJ 08855-1331, United States, 2008, pp. 768 - 773. [Online]. Available: http://dx.doi.org/10.1109/INDIN.2008.4618205

[4] K. Yliniemi, "Fault detection in district heating substations," Luleå University of Technology, Luleå, Sweden, Licentiate Thesis, ISSN 2005:60, 2005.

[5] J. Wollerstrand, P. Ljunggren, and P.-O. Johansson, "Optimal reglering av radiatorsystem," Svensk Fjärrvärme, Stockholm, Sweden, Fjärrsyn Rapport 2007:6, 2007. 
[6] J. Gustafsson, "Distributed wireless control strategies for district heating substations," Luleå University of Technology, Luleå, Sweden, Licentiate Thesis, 2009.

[7] "Embedded internet system technology botnia ab," http://www.eistec.se/, January 2009

[8] J. Johansson, M. Völker, J. Eliasson, Å. Östmark, P. Lindgren, and J. Delsing, "MULLE:a minimal sensor networking device - implementation and manufacturing challenges," in Proc. IMAPS Nordic 2004. IMAPS, 2004.

[9] "Analog devices, blackfin processors," http://www.analog. com/en/embedded-processing-dsp/blackfin/content/index.html, January 2009. [Online]. Available: http://www.analog.com/en/ embedded-processing-dsp/blackfin/content/index.html

[10] "Bluetooth Special Interest Group," http://www.bluetooth.org/, Bluetooth SIG, 2008.

[11] J. Eliasson, "Low-power Design Methodologies for Embedded Internet Systems," Ph.D. dissertation, Luleå University of Technology, SE-971 87, Luleå, Sweden, April 2008, iSSN 1402-1544.

[12] "Web Services for Devices," http: \\www.ws4d.org, January 2009.

[13] Wikipedia, "Device Profile for Web Services," http://en.wikipedia.org/ wiki/DPWS, January 2009

[14] “OPC Foundation,” http://www.opcfoundation.org, January 2009.

[15] "Bonjour - automatic discovery of computers, devices and services," http://developer.apple.com/networking/bonjour, May 2008.

[16] S. Koeppel and D. Ürge-Vorsatz, "Assessment of policy instruments for reducing greenhouse gas emissions assessment of policy instruments for reducing greenhouse gas emissions from buildings," United Nation Environment programme, Tech. Rep. ISBN: 978-963-87714-0-7, September 2007. 